\title{
Nano-scale mapping of mechanical properties in mollusk shells.
}

\author{
H. Drezner ${ }^{1}$, D. Shilo ${ }^{1}$, A. Dorgoyand ${ }^{1}$ E. Zolotoyabko ${ }^{2}$ \\ ${ }^{1}$ Department of Mechanical Engineering, Israel Institute of Technology, Haifa, 32000, Israel \\ ${ }^{2}$ Department of Materials Engineering, Israel Institute of Technology, Haifa 32000, Israel
}

Nacre, also known as mother-of-pearl, is a hard biological composite found in the inside layer of many shells and composed of $~ 95 \%$ mineral (aragonite) and $~ 5 \%$ protein-rich organic material [1]. Amazingly, although the mineral is a very brittle material, nacre can still sustain significant inelastic deformation and exhibit toughness 2-3 orders of magnitude larger than that of the mineral alone [1,2]. Numerous studies have been conducted with an aim of understanding the origin for the superior properties of the nacre and mimicking them. Recent theoretical and experimental mechanic studies show that the high toughness is a result of a large crack process zone induced by sliding of interfaces [3-5].

We apply nanoscale modulus mapping method, for visualizing the 2D-distribution of the mechanical characteristics in samples which were cut across two perpendicular directions from Perna canaliculus shells. Measured elastic moduli of the aragonite were in good agreement with bulk properties. Moreover, we found that the aragonite elastic moduli are anisotropic and we explained it by taking x-ray diffraction measurements which indicate on a strong preferred orientation of the aragonite crystalline tablets within the nacre. Further analysis was conducted by comparing the measured results with finite element simulations, which took into account the tip radius of curvature and the physical thickness of the organic layers as measured by means of Electron Back Scattered Diffraction (EBSD). This analysis showed that the Young modulus of the organic material is significantly higher than previous assumptions and is about $40 G P a$. The measured elastic moduli across mineral/organic interfaces exhibit gradual changes within a thickness at least 3 times larger than that of the EBSD and the simulations.

We explain this phenomenon to be a result of organic molecules that penetrate into the mineral material, having relatively high concentration in the vicinity of the organic/mineral interface. Thus, the nacre should be considered as a natural functionally graded material. A behavior of this type is unique to biogenic materials and distinguishes it from synthetic composite materials. Basing on these finding we suggest a mechanism for massive plastic deformation through interface sliding, which may explain their superior fracture toughness.

\section{References}

1 M. Sarikaya, I. A. Aksay, Biomimetics, Design and Processing of Materials. Polymers and

Complex Materials (Woodbury, New York , 1995)

2 S. Kamat, X. Su, R. Ballarini, A. H. Heuer, Nature 405, 1036 (2000)

3 H. Tang, F. Barthelat, H.D. Espinosa, J. Mech. Phys. Solids 55, 1410 (2007)

4 F. Barthelat, H.D. Espinosa, Experimental Mechanics 47, 311 (2007)

5 F. Barthelata, H. Tang, P.D. Zavattieri, C.-M. Li, H.D. Espinosa, J. Mech. Phys. Solids 55, 306 (2007) 